\title{
Stenting of the arterial duct combined with banding of the pulmonary arteries and atrial septectomy or septostomy: a new approach to palliation for the hypoplastic left heart syndrome
}

\author{
John L Gibbs, Christopher Wren, Kevin G Watterson, Stewart Hunter, J R Leslie Hamilton
}

\begin{abstract}
Objective-To assess the feasibility of pulmonary artery banding, atrial septectomy or septostomy, and percutaneous stenting of the arterial duct in babies with the hypoplastic left heart syndrome. Patients-Four infants with hypoplastic left heart syndrome.

Setting-Two supraregional paediatric cardiac centres.

Methods-Ductal patency was maintained initially with prostaglandin $E$. Banding of the proximal branch pulmonary arteries was performed through a median sternotomy and open atrial septectomy was performed if balloon septostomy was not. Stainless steel stents (Johnson \& Johnson) mounted in a balloon catheter were implanted into the arterial duct under radiographic control and expanded to a diameter of approximately $8 \mathrm{~mm}$, prostaglandin treatment was then stopped.
\end{abstract}

Results-All the patients survived the immediate postoperative period and maintenance of wide ductal patency was achieved in three of the four patients by stent implantation. Two weeks after the procedure two babies died of right ventricular failure and respiratory infection: some distal ductal constriction had occurred in one where the stent was not quite sufficiently distally placed. One child was discharged home 15 days after treatment and was well at follow up at age 16 weeks and one was stable but required diuretic therapy five weeks after the procedure.

Conclusions-This new approach is technically feasible. At least in the short term it seems to offer hope of effective palliation for the hypoplastic left heart syndrome and it warrants further study.

(Br Heart f 1993;69:551-555)

Attempts to develop palliative treatment for the hypoplastic left heart syndrome have met with only limited success. ${ }^{1}$ Cardiac transplantation offers some prospect of effective palliation but remains an unsatisfactory approach in the neonatal period because donor organs suitable for neonates are in short supply. ${ }^{2}$ Neonatal cardiac transplantation is further complicated by high pulmonary vascular resistance and the consequent risk of early failure of a transplanted right ventricle from an older donor. In the light of early studies of maintenance of ductal patency in lambs ${ }^{3}$ and in neonatal pulmonary atresia ${ }^{4}$ by implantation of balloon implantable steel stents and the development of stents of a suitable size for use in duct dependent systemic circulation, we elected to attempt palliation for the hypoplastic left heart syndrome by an approach that combined surgery and interventional catheterisation - that is, banding the pulmonary arteries, creation of an atrial septal defect, and stenting the arterial duct.

\section{Patients and methods}

Four neonates (birth weights $3 \cdot 0,3 \cdot 7,4 \cdot 1$, and $3.3 \mathrm{~kg}$ ) presented in the first week of life with heart failure caused by mitral and aortic atresia with hypoplasia of the left ventricle. Their parents opted for an attempt at palliation. The babies were treated with intravenous prostaglandin $\mathrm{E}$ to maintain duct patency.

\section{SURGERY}

Three patients underwent the surgical stage of treatment first, the fourth had the surgical stage of treatment after balloon atrial septostomy and ductal stenting. Surgery was undertaken at the ages of $34,22,19$, and 11 days respectively. A median sternotomy was performed and the proximal right and left pulmonary arteries were banded with nylon tape (coated with Silastic in three cases), each band was held in place by two Ethibond $5 \cdot 0$ sutures; we attempted to reduce the pulmonary artery diameters by approximately $50 \%$. Open atrial septectomy was performed in the first two babies during a brief (approximately 10 minute) episode of circulatory arrest after cooling to $20^{\circ} \mathrm{C}$ on cardiopulmonary bypass. The operative procedure was similar in each child, but in the second case the duct was dilated peroperatively with a 10 $\mathrm{mm}$ diameter balloon catheter inserted through the pulmonary trunk because of signs of ductal constriction.

\section{CARDIAC CATHETERISATION AND STENT} IMPLANTATION

We chose to use balloon expandable PalmazSchatz stainless steel biliary stents (Johnson \& 
Johnson) because of their suitable size. The stent is $20 \mathrm{~mm}$ long and consists of two slotted steel tubes approximately $9 \mathrm{~mm}$ long, joined by a single bridge. The bridge joining the two sections can be carefully cut to produce two shorter stents $9 \mathrm{~mm}$ long, though this is not recommended by the manufacturer. Though designed to be expanded to 6 $\mathrm{mm}$, the stent can be dilated to $10 \mathrm{~mm}$ with an appropriate balloon catheter. The full length stent shortens by approximately $3 \mathrm{~mm}$ after expansion to $8 \mathrm{~mm}$ diameter. Ductal stenting was performed under general anaesthetic in the four babies at $25,26,24$, and 4 days of age respectively. The left femoral artery and the right femoral vein were catheterised with French 4 and French 5 multipurpose catheters respectively. Heparin (100 units $/ \mathrm{kg}$ ) was given intravenously. Balloon atrial septostomy was performed immediately before stent implantation in the two patients who did not have surgical septectomy. Pulmonary angiography in lateral projection was used to delineate the ductal anatomy, and in both anteroposterior and lateral projections to assess pulmonary artery band siting when appropriate (fig 1). We estimated the length of the duct with digital software callipers calibrated to the catheter diameter: it was approximately $12 \mathrm{~mm}, 7$ $\mathrm{mm}, 13 \mathrm{~mm}$, and $15 \mathrm{~mm}$ in the four cases. The diameters of the ducts ranged from approximately $4 \mathrm{~mm}$ to $6 \mathrm{~mm}$ at the aortic end and from $6 \mathrm{~mm}$ to $8 \mathrm{~mm}$ at the pulmonary artery end. The aortic catheter was advanced to the level of the duct for use as a landmark. An exchange guide wire $(0.035$ inch) was advanced via the right heart catheter from the pulmonary artery through the duct into the distal descending aorta and the French 5 multipurpose catheter was exchanged for a French 7 curved sheath (Cordis) which was advanced to the descending aorta just distal to the duct. Half length stents were used in three cases and a full length stent in one. The appropriate length stent was mounted on a French $5,20 \mathrm{~mm}$ long compliant balloon catheter (Cordis, Opta 5). The final diameter of this balloon

Figure 1 Pulmonary angiography in case $1(A)$ in the anteroposterior projection, with the site of the right pulmonary artery band arrowed and $(B)$ in the lateral projection, with the site of the left

pulmonary artery band arrowed. In the lateral projection the arterial duct, measured as the distance between (small double arrows) the level of the arrows) the level of the
origin of the left pulmonary artery and the ductal junction with the aorta, was about $12 \mathrm{~mm}$ long. $P A$ main pulm $\mathrm{P} A$, main pulmonary artery; Ao, descending aorta. ranged from $6.6 \mathrm{~mm}$ to $8.3 \mathrm{~mm}$, depending on the inflation pressure used. The stent was advanced to the distal part of the sheath and the sheath was then withdrawn into the main pulmonary artery to leave the stent exposed in the duct. The stent was deployed in the distal part of the duct (guided by hand injec- 므 tions of contrast through the aortic catheter) $\frac{I}{\Phi}$ by inflation of the balloon to $10 \mathrm{~atm}$ for 10-15 seconds, resulting in a balloon diame- $\stackrel{.}{.}$ ter of approximately $7 \cdot 7 \mathrm{~mm}$. Satisfactory $\ddot{\vec{F}}$ stent placement was assessed by repeat $\stackrel{?}{\rightarrow}$ pulmonary angiography. In the two cases in which ductal length necessitated a second $\frac{\bar{F}}{\overline{5}}$. stent the procedure was repeated, the second $\frac{\vec{\sigma}}{\sigma}$ (half length) stent being delivered to the $\varrho$ proximal part of the duct and deployed at 10 \% atm pressure, with its distal part tucked inside $\overrightarrow{0}$

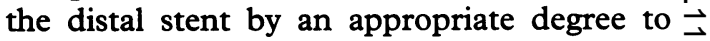
tailor the overall stent length to the individual $\vec{\sigma}^{\circ}$ ductal anatomy. The prostaglandin infusion was stopped and final pulmonary angiography $\stackrel{\circ}{\circ}$ was performed to confirm appropriate stent positioning and ductal patency.

The patients were electively ventilated and $\stackrel{\circlearrowleft}{\rightarrow}$ monitored on the intensive care unit. 의 Antibiotic treatment (cefuroxime) was given prophylactically for 48 hours. Anticoagulation $£$ by heparin infusion was continued to keep $\bar{\nabla}$ the activated partial thromboplastin time at $\vec{\varphi}$ between 2 and 2.5 times the control level, $\stackrel{\varrho}{\omega}$ pending stabilisation on warfarin. We elected to continue anticoagulation for one month, anticipating that endothelialisation of the stent would be well advanced by then. Antiplatelet treatment was not given.

\section{Results}

Loud systolic murmurs caused by the pulmonary artery bands were present in each case after the surgical stage of treatment. The first child required 48 hours' ventilation and was clinically considerably improved after 3 surgery but we were unable to wean the sec- $\dot{\delta}$ ond and third patients from ventilation because of poor right ventricular function and 0 respiratory infection. The fourth patient remains haemodynamically stable with excel-o

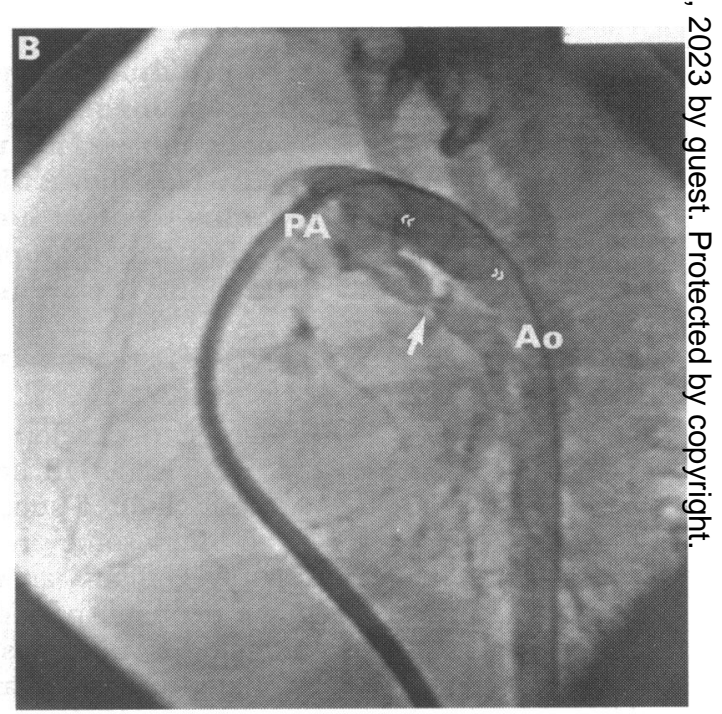


lent right ventricular function but requires diuretic treatment because of relatively high pulmonary blood flow.

Ductal patency was maintained with prostaglandin $\mathrm{E}$ without difficulty in the first case after the surgical stage of treatment, allowing ductal stenting to be planned electively. In the second case stent implantation was prompted by clinical signs of recurrent ductal constriction requiring very high dose intravenous prostaglandin after surgical treatment (which in this case had included balloon dilation of the duct). Balloon septostomy was performed without difficulty in the two patients in whom it was attempted. The size of the left atrium in each case, however, limited the inflation size of the balloon. We were able to pull balloons ( $4 \mathrm{ml}$ Fogarty) filled to $3 \mathrm{ml}$ and $2.5 \mathrm{ml}$ through the respective foramens but the balloons failed to cross the septum when further inflated. The difference in mean atrial pressures before and after septostomy in the two cases were $7 \mathrm{~mm} \mathrm{Hg}$ falling to $5 \mathrm{~mm} \mathrm{Hg}$ and $17 \mathrm{~mm} \mathrm{Hg}$ falling to $5 \mathrm{~mm} \mathrm{Hg}$.

Pulmonary angiography in the three cases who underwent catheterisation after surgery showed the band positions with balanced perfusion of the lungs (fig 1A) in cases 1 and 3 but with preferential blood flow to the left lung with apparent occlusion of the right upper lobe pulmonary artery in case 2 .

Stent deployment was accompanied on all occasions by bradycardia, extreme hypotension, and ST segment elevation on the electrocardiogram. This spontaneously recovered over one to two minutes. A further prolonged episode of ST segment elevation with bradycardia, atrioventricular dissociation, and extreme hypotension occurred in the first case after final pulmonary angiography. This patient required cardiac massage, intravenous adrenaline and calcium, and temporary pacing. Sinus rhythm and systolic blood pressure of $80 \mathrm{~mm} \mathrm{Hg}$ were restored within five minutes. In the third case we elected to stent the duct with two overlapping half length stents. Both stents were satisfactorily deployed, but

Figure 2 Final radiographic appearances of the ductal stent in case 4 in anteroposterior $(A)$ and lateral (B) projections. withdrawal of the balloon catheter after the second delivery resulted in displacement of the proximal stent into the pulmonary trunk with the guide wire still through it. The stent was remanoeuvred into the proximal duct and redilated with a $10 \mathrm{~mm}$ diameter balloon, but again became displaced into the pulmonary trunk when the balloon was withdrawn. We were able to remanoeuvre the stent back into the duct, in a stable position but with its long axis lying diagonally in relation to the distal stent. This position was accepted because the patient remained haemodynamically stable. Our previous experience showed that coronary artery stents are difficult to see clearly on radiographic screening ${ }^{4}$ but the larger biliary stents used in these patients with duct dependent systemic circulation were relatively easily seen on screening and were plainly visible on chest radiographs postoperatively (fig 2 ).

The first child was extubated four hours after the procedure and returned to the ward the following morning. He was discharged home two weeks later, taking warfarin and diuretics. He remained slightly tachypnoeic but with normal femoral pulses and a systolic murmur related to the pulmonary artery bandings and he was well and gaining weight at follow up at the age of 16 weeks. The second and third patients remained ventilator dependent with clinical and echocardiographic signs of right ventricular failure and died 12 days and 14 days respectively after ductal stenting. Both ducts were patent at necropsy, but in one the stent fell 2-3 mm short of the ductal junction with aorta and some distal ductal constriction had occurred. The fourth child remains in hospital and is steadily improving five weeks after the completed procedures with normal femoral pulses but with breathlessness due to relatively high pulmonary blood flow.

\section{Discussion}

SURGERY

The surgical stage of treatment, which included a short period of cardiopulmonary

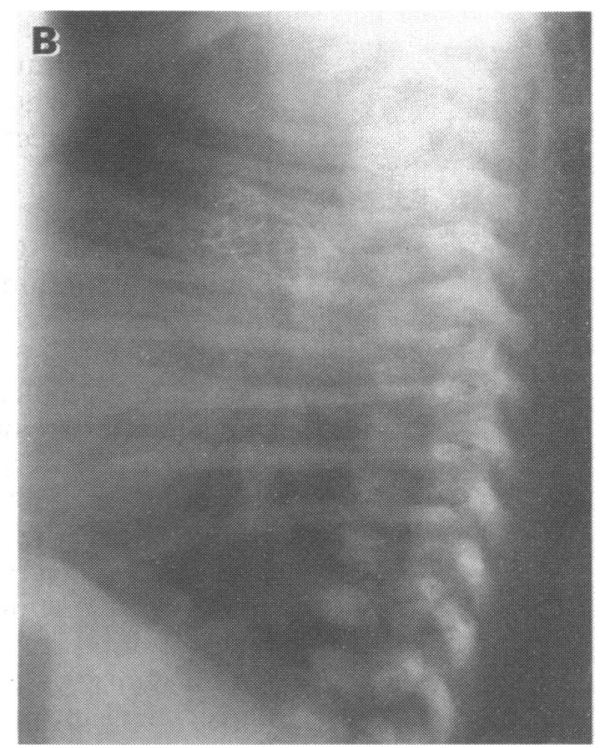


bypass in two cases, was well tolerated and uneventful in three of the patients and pulmonary angiography showed well balanced flow to the lungs in the two who were catheterised after surgery. Surgery in the second child was less well tolerated due, at least in part, to ductal constriction. Postoperative ductal patency was achieved in this patient only by giving very large doses of intravenous prostaglandin $\mathrm{E}(0.5 \mu \mathrm{g} / \mathrm{kg} / \mathrm{min}$, ten times the dose used initially in this child). Peroperative balloon dilatation of the arterial duct had no more than a very brief beneficial effect in this patient and was followed by aggressive ductal constriction, a finding that accords with our earlier experience with pulmonary atresia ${ }^{4}$ but contrasts with the experience of others who have reported some success in delaying ductal closure by balloon dilatation alone. ${ }^{5}$ Well balanced pulmonary artery banding proved particularly difficult in one of our patients and pulmonary angiography later showed preferential flow to the left lung, with the right upper lobe branch apparently occluded by the right-sided band in this child. We are optimistic that further experience will improve the results of bilateral band placement. The bands were coated with Silastic in three cases in the hope that this might facilitate later removal, and were held in place only by two fine sutures, again in the hope of facilitating later band removal or balloon angioplasty.

\section{CREATION OF AN ATRIAL SEPTAL DEFECT}

An elective decision was taken to opt for open septectomy in two of our patients in whom the left atrium was small; balloon septostomy was performed in the two cases where the left atrium was reasonably well developed. Though it proved possible to perform open septectomy in both patients with only very short periods of circulatory arrest, this was poorly tolerated in our second patient and it is clear that cardiopulmonary bypass should be avoided in this condition if at all possible. However, when the left atrium is small, as it often is in hypoplastic left heart syndrome, it may be impossible to achieve an adequate interatrial communication by closed septectomy or balloon septostomy. Further follow up will be required to assess the adequacy of the interatrial communication after balloon septostomy and whether further procedures to enlarge the defect will become necessary as the child grows.

\section{DUCTAL STENTING}

Stent implantation was technically a straightforward procedure in three patients, no doubt partly because of our earlier experience with the more complex ductal anatomy in pulmonary atresia. ${ }^{4}$ In one patient a half length stent became displaced from the proximal part of the duct to come to lie on the guidewire in the pulmonary trunk. In this case the proximal duct diameter closely approached that of the deployed stent diameter and in retrospect we think that a full length stent would have been anchored by the narrower distal part of the duct. At necropsy in this patient the stent position was not ideal and there was some distal ductal stenosis; the proximal stent was lying at the junction of the pulmonary trunk and the duct and was still poorly aligned with the distal stent but there were no apparent ill effects from this. Judging the deployed stent length in relation to the w ductal length is an important factor in choos- $I$ ing both the length of stent to be implanted and the exact position of the stent before deployment. This judgement is complicated $\overrightarrow{\vec{F}}$ by progressive shortening of the stent with $\stackrel{\vec{P}}{+}$ increasing dilatation, a factor which almost certainly played a major part in our error in $\frac{\bar{D}}{\bar{c}}$ placing the stent slightly too proximally in the patient who was later found to have devel- $\square$ oped distal duct constriction. We are optimistic that our judgement will improve with $\overrightarrow{0}$ increasing experience. However, even with $\overrightarrow{\vec{H}}$ the use of digital callipers it proved remark- $\vec{\omega}$ ably difficult to obtain an accurate measurement of ductal length, mainly because 7 accurate calibration of the callipers is difficult to achieve. We believe that it is probably best of to err by implanting too short a stent initially $\stackrel{G}{-}$ because a second stent can be implanted and $\frac{\rho}{5}$ telescoped into the first to tailor overall stent length to individual duct anatomy. Though $\overleftarrow{c}$ blood can flow easily through the lattice of $\frac{\bar{\sigma}}{\sigma}$ the expanded stent, we thought it important $\vec{\bullet}$ in the setting of the hypoplastic left heart syn- $\circlearrowright$ drome that the stent should not protrude into the aortic lumen because of the theoretical risks of interference with coronary arterial flow, of coronary or cerebral emboli arising from the stent, or of haemolysis. Despite $\frac{}{\varnothing}$ these fears, however, poor stent alignment in our third case was not associated with any apparent untoward consequences.

Speed seems to be an important factor to 总 minimise complications in this procedure. A $\frac{\dot{\partial}}{\vec{\sigma}}$ quick procedure will reduce the attendant risk of hypothermia in the neonate in the catheter laboratory and will minimise haemodynamic upset caused by passage of the stiff combination of a French 7 sheath, a balloon catheter, a stent, and a guidewire through the right side of the heart. This clearly interferes with cardiac function and a heart with hypoplasia of the left elements is likely to be particularly sensitive to such interference. We believe that o the haemodynamic complications in our first $N$ patient were related partly to such splinting of $\underset{\omega}{N}$ the heart by the delivery system and partly to 0 the toxic effect of angiographic contrast on 0 myocardial perfusion. Our experience now suggests that ductal stenting should take no $\stackrel{?}{+}$ more than one hour if a single stent is implanted. Contrast dosage should be conscientiously kept to the minimum necessary for accurate stent positioning.

\section{POSTOPERATIVE CARE}

Except for anticoagulation no specific postoperative measures were used after completion of the treatment. It is not clear whether anti- $\stackrel{\rightleftharpoons}{\rightleftharpoons}$ coagulation is really necessary in the presence of high flow through a steel stent, but the clear potential for any thrombus to embolise to the coronary or cerebral circulation led us 
to opt for anticoagulation for one month, by which time we anticipated that endothelialisation would be well advanced.

\section{FUTURE TREATMENT}

If adequate early palliation is achieved by this procedure cyanosis due to gradual reduction in flow through the banded pulmonary arteries is likely to occur at some time during the first few years. With the banding technique we used it may prove possible to improve pulmonary flow by later balloon angioplasty. Failing this, or if right ventricular function deteriorates, the possibilities of cavopulmonary anastomosis or cardiac transplantation will need to be explored. Attempted transplantation at this later stage might carry a greater chance of a donor organ becoming available, though surgery would be complicated by the need to deband the pulmonary arteries. It seems likely that a ductal diameter of nearly $8 \mathrm{~mm}$ will prove sufficient to allow unobstructed systemic blood flow for many months. Should the ductal diameter prove restrictive, however, it may well be possible to redilate the stent later. ${ }^{6}$

Our preliminary experience with this new approach of combined pulmonary artery banding, creation of an atrial septal defect, and ductal stenting suggest that it shows promise in providing effective palliative treatment for the hypoplastic left heart syndrome and that it warrants further study.

1 Pigott JD, Murphy JD, Barber G, Norwood WI. Palliative reconstructive surgery for hypoplastic left heart synreconstructive surgery for hypoplastic

2 Stuart AG, Wren C, Sharples PM, Hunter S, Hey EN. Hypoplastic left heart syndrome: more potential transplant recipients than suitable donors. Lancet 1991 337:957-9.

3 Coe JY, Olley PM. A novel method to maintain ductus arteriosus patency. $\mathcal{f} \mathrm{Am}$ Coll Cardiol 1991;18:837-41.

4 Gibbs JL, Rothman MT, Rees M, Parsons JM, Blackburn ME, Ruiz CE. Stenting of the arterial duct: a new approach to palliation for pulmonary atresia. Br Heart $\mathcal{f}$ 1992;67:240-5

5 Walsh KP, Sreeram N, Franks R, Arnold R. Balloon dilation of the arterial duct in congenital heart disease. Lancet 1992;339:331-2.

6 Coe JY, Timinsky J, McKendrick R, Tio FO, Olley PM. Redilating ductal stents [abstracts]. Circulation 1991;48: II-545. 